\title{
Taylor-Couette flow in a two-layer stratified fluid: instabilities and mixing
}

\author{
E.V. Ermanyuk ${ }^{1}$, J.-B. Flór* \\ Laboratoire des Ecoulements Geophysiques et Industriels, INPG-CNRS-UJF, BP53X, \\ Grenoble Cedex 9 38041, France
}

In this preliminary experimental study we investigate the mixing of a salt-stratified two-layer fluid in a Taylor-Couette flow. We focus on two flow regimes, one in which waves are present in the pycnocline and vortices in the homogeneous layers, and one with vortices also in the pycnocline. The transition between these two regimes differs significantly compared to the wave-vortex transition found in a uniform stratification, investigated by Caton et al. [Caton, F., Janiaud, B., Hopfinger, E.J., 2000. Stability and bifurcations in stratified Taylor-Couette flow. J. Fluid Mech. $419,93-124]$, and is accompanied by a larger jump in fundamental frequency of the density fluctuations.

Most density gradients show a strong tendency to sharpen with time, with layer formation in the case of an initially thick interface. On a long time-scale, the entrainment of a smooth pycnocline shows cascade-like bifurcations before its transition to chaos. In the latter case, a decrease of the initial density gradient is observed.

We discuss the mixing efficiency, measured by the Richardson flux number $R i_{\mathrm{f}}$. Its value is a function of the Reynolds number $\sim R e^{k}$, with $k=3 / 2$, and for Reynolds numbers $\mathrm{O}\left(10^{3}\right)$, the mixing efficiency is of order $\mathrm{O}\left(10^{-2}\right)$.

Keywords: Taylor-Couette flow; Stratified rotating flows; Mixing; Pycnocline

\footnotetext{
* Corresponding author. Fax: +33 476825721.

E-mail address: flor@hmg.inpg.fr (J.-B. Flór).

${ }^{1}$ Permanent address: Lavrentyev Institute of Hydrodynamics, 630090 Novosibirsk, Russia.
} 


\section{Introduction}

Mixing in density stratified fluids plays an important role in numerous geophysical and engineering applications, and is responsible for the transport of heat, nutrients and pollutants in the oceans and atmosphere, as well as in various technical devices. The driving mechanisms for mixing in oceanographic processes have been thoroughly discussed in the literature over the past five decades (see Kato and Phillips, 1969; Linden, 1979; Fernando, 1991; Staquet and Sommeria, 2002, Caulfield and Peltier, 2002). Typical experiments on mixing include the entrainment and overturning motion in shear flows (e.g., Strang and Fernando, 2001), stirring by different mechanical devices such as oscillating grids, or entrainment of vortex rings (Linden, 1973, for a review see Fernando, 1991).

In many geophysical flows, mixing is produced by anisotropic flows having a significant horizontal vorticity component. For example, Langmuir circulation, which is characterized by the occurrence of organized horizontal helical vortex-structures with their axis directed along the wind-induced main flow, is thought to play a significant role for the mixing of the ocean pycnocline (Garrett, 1996). Motivated by this application, we investigate the evolution of an interface - or pycnocline — of a two-layer fluid in the presence of Taylor vortices. Taylor-Couette flows are extensively investigated for homogeneous fluids and give a controlled access to different flow regimes, ranging from wavy flows, wavy-vortex flows and turbulence (see Andereck et al., 1986). The extension to Taylor-Couette flows with linear axial stratification have been investigated experimentally and theoretically by Hopfinger and co-workers (see Boubnov et al., 1995; Boubnov and Hopfinger, 1997; Caton et al., 2000) and numerically by Hua et al. (1997) and new regimes are shown to occur as a consequence of the different dynamics. Stratification increases the threshold rotation-rate, $\Omega_{\mathrm{c}}$, for the onset of the Taylor vortices, while for small rotation-stratification frequency ratio, $\Omega / N$, the Taylor vortices are flattened. In the two-layer stratification, the dynamics are more complicated, since the buoyancy frequency, $N$, is not constant, and flow properties may vary locally. Waves generated inside the pycnocline find critical layers above and beneath, leading to reflection or absorption, while vortices outside the pycnocline perturb the stratified layer and the motion there in. These effects may alter the dynamics as well as the regimes found in linearly stratified TC-flows and have not been investigated before. In the next section we present our experimental setup, followed by the results on the different observed regimes in Section 3.1 and the mixing in Section 3.2. We briefly discuss the results in Section 4.

\section{Experimental setup and procedure}

\subsection{Apparatus}

The experimental apparatus consists of two Plexiglas coaxial cylinders with radii, respectively, $a=15 \mathrm{~cm}$ and $b=20 \mathrm{~cm}$, and is an enlarged version of the stratified Taylor-Couette system described in Caton et al. (2000) further referred to as CJH. The outer cylinder is fixed while the inner cylinder is driven by a DC-motor at a rotation rate $\Omega$ of a value between 0.08 and $0.56 \mathrm{~s}^{-1}$. The total depth of the fluid in the experiments, $H$, is $76 \mathrm{~cm}$ yielding a moderate 
value of aspect ratio $A=H / d=15.2$, where $d=b-a$. The annular space between the cylinders was filled with a two-layer salt-stratified fluid and tap-water is used as working fluid. The vertical density distribution can be approximated as

$$
\rho(z)=\rho_{0}\left[1-\frac{\epsilon}{2} \tanh \frac{2(z-h)}{\delta}\right], \quad \text { where } \rho_{0}=\frac{\rho_{1}+\rho_{2}}{2} \text { and } \epsilon=\frac{\rho_{2}-\rho_{1}}{\rho_{0}} \text {, }
$$

with $z=0$ at the free surface and the $z$-axis directed vertically upwards; $h$ is the depth of the upper fluid (in experiments $h=H / 2$ ), $\delta$ the characteristic thickness of the pycnocline, and $\rho_{1}, \rho_{2}$ and $\rho_{0}$ are the fluid densities in the upper and lower layers and mean density, respectively. Experiments with a smooth pycnocline were performed at large $\delta$, obtained after having the interface diffused during several days.

The density difference between the layers was varied within the range $0.0015 \leq \epsilon \leq$ 0.02 . In the otherwise quiescent fluid, parameter $\delta$ changed with time $t$ as $\delta \sim t^{1 / 2}$, owing to the molecular diffusion of salt. Both, sharp and smooth pycnoclines were studied with typical

Table 1

Governing parameters of the experimental runs, where series $A$ and $B$ and experiment $C$ correspond to runs with an initially smooth interface whereas in series $D, E$ the interface was initially sharp.

\begin{tabular}{|c|c|c|c|c|c|c|}
\hline Experiment series & $\epsilon$ & $\delta / d$ & $\Omega\left(\mathrm{s}^{-1}\right)$ & $N\left(\mathrm{~s}^{-1}\right)$ & $T a \times 10^{5}$ & $G r \times 10^{6}$ \\
\hline A & 0.006 & 1.7 & $\begin{array}{l}0.068 \\
0.084 \\
0.098 \\
0.111 \\
0.125 \\
0.139 \\
0.154 \\
0.165\end{array}$ & 0.83 & $\begin{array}{l}1.5 \\
2.3 \\
3.1 \\
3.9 \\
5.0 \\
6.2 \\
7.6 \\
10.6\end{array}$ & 4.3 \\
\hline B & 0.024 & 1.56 & $\begin{array}{l}0.097 \\
0.123 \\
0.151 \\
0.178 \\
0.209 \\
0.268 \\
0.293 \\
0.33\end{array}$ & 1.74 & $\begin{array}{l}3.0 \\
4.8 \\
7.3 \\
10.1 \\
14.0 \\
23.0 \\
27.3 \\
34.9\end{array}$ & 19 \\
\hline $\mathrm{C}$ & 0.006 & 1.7 & 0.16 & 0.83 & 8.2 & 4.3 \\
\hline $\mathrm{D}$ & $\begin{array}{l}0.0015 \\
0.003 \\
0.006 \\
0.009 \\
0.02\end{array}$ & 0.2 & 0.5 & $\begin{array}{l}1.21 \\
1.72 \\
2.43 \\
2.97 \\
4.43\end{array}$ & 80 & $\begin{array}{l}9.3 \\
18.5 \\
37.5 \\
55 \\
123\end{array}$ \\
\hline $\mathrm{E}$ & 0.003 & 0.2 & $\begin{array}{l}0.29 \\
0.35 \\
0.41 \\
0.46 \\
0.53\end{array}$ & 1.72 & $\begin{array}{l}27 \\
39 \\
54 \\
68 \\
90\end{array}$ & 18.5 \\
\hline
\end{tabular}

The Taylor number, $T a$, is defined as $\frac{4 \Omega^{2}}{Y^{2}} \cdot d^{4} /\left(b^{2} / a^{2}-1\right) \approx 3.2 \times 10^{7} \Omega^{2}$ and the Grashof number $G r=$ $N^{2} d^{4} / \nu^{2}=6.25 \times 10^{6} N^{2}$ with $N=(g \epsilon / \delta)^{0.5}$. 
values $\delta / d$ around 0.2 and 2, respectively. The Reynolds number $R e=\Omega a d / v$ (where $v$ is the kinematic viscosity) was varied between 600 and 4200. A list with the experimental parameters is given in Table 1, which includes the experimental values of the Taylor number and Grashof number.

For an identification of the observed flow patterns, we used the shadow-graph technique, which visualizes variations in the second spatial derivative of the instantaneous density profile by light and dark projections of a punctual light-source that shines through the fluid. The white and dark-gray band represents the interface (see Fig. 1a). These light intensity fluctuations were recorded with a B\&W analog camera. From the images a fixed vertical line of pixels was selected in real time and added with a prescribed sampling rate to the stack of lines taken at previous time steps. This vertical line of pixels was taken at $1 \mathrm{~cm}$ distance from the wall of the outer cylinder (see arrows in Fig. 1a and Fig. 2a). For the measurement of mixing across the density interface we used laser-induced fluorescence.
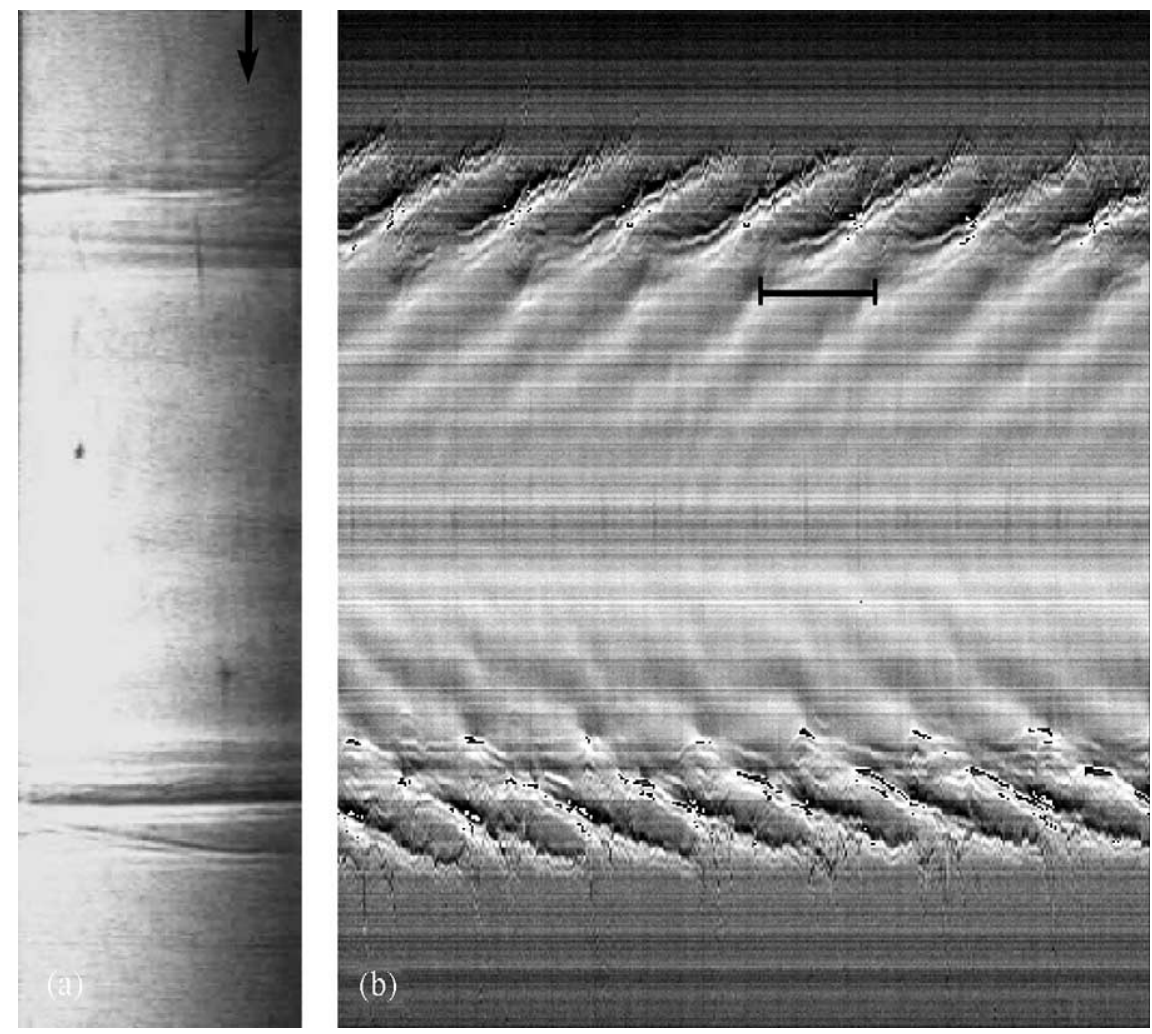

Fig. 1. Shadow-graph image of the gap between the cylinders (a) and (b) spatio-temporal image of the selected vertical line of pixels in the wave-vortex regime. The picture contains 674 vertical lines taken at the acquisition frequency $1 \mathrm{~Hz}$, and time increases from left to right. Experimental parameters: $\epsilon=0.024, \delta / d=1.56, \Omega=$ $0.151 \mathrm{rad} / \mathrm{s}$. 

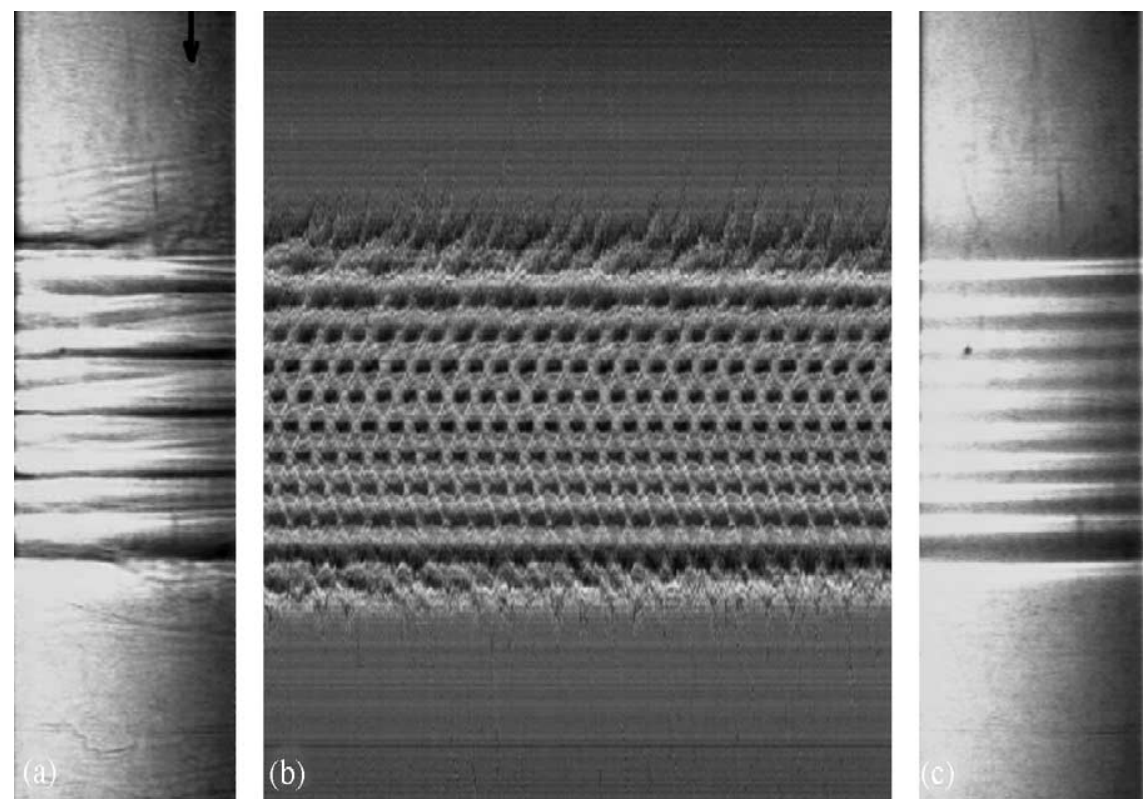

Fig. 2. Typical shadow-graph image of the gap between the cylinders (a), (b) spatio-temporal image of the selected vertical line of pixels for a total acquisition time of $443 \mathrm{~s}$ at the sampling frequency $1 \mathrm{~Hz}$, and (c) shadow-graph image of the gap between the cylinders after the experimental run. Experimental parameters $\epsilon=0.024, \delta / d=1.56$, $\Omega=0.268 \mathrm{rad} / \mathrm{s}$.

\section{Experimental results}

\subsection{Flow regimes and entrainment in a smooth pycnocline}

We have noted two different flow regimes depending on the rotation rate while keeping the stratification of the interface constant. For low rotation values, Taylor vortices are present in the two homogeneous layers, while the flow is stabilized and axisymmetric in the centre of the pycnocline. Since the buoyancy frequency decreases to zero at the edges of the pycnocline, waves are generated at some level above the pycnocline centre. This regime is referred to as the wave-vortex regime. For higher rotation rates, vortices are present, both in the pycnocline and the two homogeneous layers. The latter is referred to as the vortex-vortex regime.

A typical example of the wave-vortex regime, is illustrated in Fig. 1a, showing highgradient interfaces on the upper and lower edges of the pycnocline, with alteration of dark and light zones inside the pycnocline. Fig. 1b shows the spatio-temporal image of the vertical line of pixels marked by the black arrow in Fig. 1a. Fig. 1b reveals the wave motion inside the pycnocline. Note that inside the pycnocline, the density varies with height. At the centre of the pycnocline where the buoyancy is maximal, waves are suppressed in agreement with results in a linearly stratified fluid by Boubnov and Hopfinger (1997), while waves with increasing amplitude occur with distance from the pycnocline-centre and decreasing 
buoyancy. These waves are progressive and propagate towards the edges of the pycnocline. The time-history of the light fluctuations inside the pycnocline yields sine curves with a well defined frequency $\Omega_{\mathrm{f}}$.

The saw-like patterns at the pycnocline edges represent the motion of the vortices, which interact with the waves that are periodically emitted in the pycnocline. The waves break above and below the pycnocline where the local buoyancy frequency is relatively small, and dense (light) fluid is entrained into the upper (lower) layer. Nevertheless little mixing takes place, and the initial density profile is quickly recovered after turning off the inner cylinder rotation. Note, that in contrast to the standing waves observed in the wavy regime by $\mathrm{CJH}$ in a linearly stratified fluid, we observe progressive wave patterns; their amplitude increases with distance from the pycnocline centre due to the decreasing buoyancy frequency, as can be inferred from the variation in wave-signature in the shadow-graph images (see Fig. 1).

For higher rotation rates the wave motion is unstable, leading to the formation of stratified vortices in the pycnocline region. The threshold rotation-rate for the occurrence of vortex motion corresponded well with that in a linear stratified fluid found by Boubnov and Hopfinger (1997) in the same device for $\Omega_{\mathrm{c}} \geq 0.075 N+0.075$, with $N$ the maximum stratification of the pycnocline. Fig. 2a shows an example of this vortex-vortex regime, consisting of a system of narrow layers separated by clearly visible interfaces. The spatiotemporal image of this flow is shown in Fig. $2 \mathrm{~b}$ for the same $\delta / d$ and $\epsilon$ as in Fig. 1 . In the pycnocline we notice periodic oscillations with upward and downward propagation direction and a well-pronounced fundamental frequency $\Omega_{\mathrm{f}}$. At the edges of the pycnocline, the noise increases and fluctuations at frequencies lower than the fundamental one appear (typically, at $\Omega_{\mathrm{f}} / 2$ and $\Omega_{\mathrm{f}} / 3$ ).

Five minutes after having turned off the rotation of the inner cylinder the shadow-graph images reveal a well-pronounced layered structure (see Fig. 2c), showing that the stratified vortices effectively mix the pycnocline. The ratio between the vertical and horizontal sizes of the layered structure in Fig. 2c is remarkably low. It reaches 1:6 in the middle of the pycnocline and slightly increases to $1: 5$ at the edges of the pycnocline, due to a decrease of the local buoyancy frequency. Note, however, that the vertical size of the structure does not increase in inverse proportion to the local buoyancy frequency. The vertical size of the layers at the edges of the pycnocline is 1.2 times greater than in the middle of the pycnocline, while the initial local buoyancy frequency at the horizons of these layers is 1.4 times smaller than in the middle of the pycnocline. Also, it should be noted that the aspect ratio of 1:6 is equal to the lowest aspect ratio observed in the same apparatus by Boubnov and Hopfinger (1997) for a uniform stratification. In a smaller Taylor-Couette device, the onset of the stratified vortex regime in the uniformly stratified fluid leads to the formation of structures having an aspect ratio between vertical and horizontal size around 1:2 (see $\mathrm{CJH})$.

The transition from the wave-vortex regime to the vortex-vortex regime is accompanied by a jump in the mean rotation frequency $\Omega_{\mathrm{f}}$ of the $3 \mathrm{D}$ flow-structure. This (fundamental) frequency can be measured from the light-intensity fluctuations shown in Fig. 1b, where one single revolution period of the flow structure around the cylinder axis is depicted by a horizontal bar. For a purely azimuthal flow the rotation frequency $\Omega_{0}$ with which the flow structure drifts can be derived for the Taylor-Couette flow, and yields after averaging over 
the volume in the gap (see CJH):

$$
\left\langle\Omega_{0}\right\rangle=\left(-\frac{a^{2}}{b^{2}-a^{2}}+\frac{2 a^{2} b^{2}}{\left(b^{2}-a^{2}\right)^{2}} \ln \frac{b}{a}\right) \Omega .
$$

For our setup this gives $\left\langle\Omega_{0}\right\rangle=0.411 \Omega$. When the axisymmetry of the system is broken by an azimuthal mode, $m \geq 1$, the flow-structure precesses relative to the mean rotation frequency with a frequency, $\alpha$, given by the relation (Knobloch, 1996; Caton et al., 1999):

$$
\Omega_{\mathrm{f}}=m\left\langle\Omega_{0}\right\rangle+\alpha(m) .
$$

As $N$ increases, the frequency jump occurs at higher $\Omega$ due to the stabilizing effect of the stratification (see Fig. 3). Both, in the wave-vortex regime and the vortex-vortex regime, $\Omega_{\mathrm{f}}$ increases linearly with $\Omega$. The least square approximation yields for the wave-vortex regime

$$
\Omega_{\mathrm{f}}=0.41 \Omega+0.0014
$$

and for the vortex-vortex regime

$$
\Omega_{\mathrm{f}}=0.81 \Omega+0.138 .
$$

In the wave-vortex regime, the dependence of $\Omega_{\mathrm{f}}$ on $\Omega$ is remarkably close to the angular velocity for purely azimuthal flow, $\left\langle\Omega_{0}\right\rangle$. Surprisingly, this result corresponds with the $\Omega_{\mathrm{f}}(\Omega)$-relation in the stratified vortex regime, characterized by counter-rotating vortex pairs with azimuthal mode $m=1$ (see $\mathrm{CJH}$ ), whereas the nature of the wave-vortex regime, with progressive waves in the pycnocline and vortical motion at the edges of the pycnocline, is altogether different. Also in both cases, the evolution of $\Omega_{\mathrm{f}}$ with $\Omega$ does not depend on stratification since the data obtained for different values of $N$ fall on the same straight line. Though further research is required to give a sound explanation, possibly, the drift and precession of the vortical motions in the weakly stratified upper and lower part of the

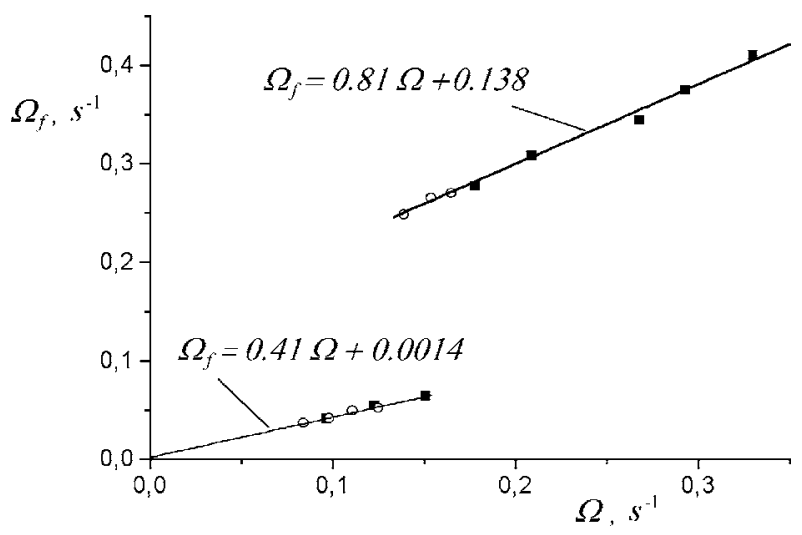

Fig. 3. Wave-vortex transition (open symbols: $\epsilon=0.006, \delta / d=1.7, N=0.83 \mathrm{rad} / \mathrm{s}$; black symbols: $\epsilon=0.024$, $\delta / d=1.56, N=1.74 \mathrm{rad} / \mathrm{s})$. 


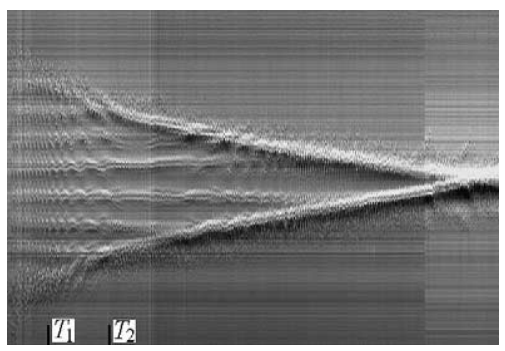

Fig. 4. Spatio-temporal shadow-graph image of entrainment in a smooth pycnocline. The acquisition frequency is $0.05 \mathrm{~Hz}$ and the total acquisition time amounts $9.8 \mathrm{~h}$. Experimental run $\mathrm{C}$ with $\Omega=0.16 \mathrm{rad} / \mathrm{s}$ and times $T_{1}=$ $2940 \mathrm{~s}, T_{2}=7320 \mathrm{~s}$ after the onset of rotation.

pycnocline govern the drift at the interface, suggesting the correspondence in fundamental frequency with the stratified-vortex regime and the precession value of exactly twice that in $\mathrm{CJH}$.

In the vortex-vortex regime, the shadow-graph lines are propagating in up- and downward directions (see Fig. 4), while from (1) and (2) we note that in the vortex-vortex regime the coefficient of $\Omega$ is twice the value of the coefficient in the wave-vortex regime, and the precession about 100 times as large. This high value of the precession suggests the presence of higher modes $(m=2)$, most likely to be expected in the layers with absent, or relatively weak stratification. At the transition in a two-layer fluid from the wave-vortex regime to the vortex-vortex regime we note a clear jump in frequency $\Omega_{\mathrm{f}}$. In contrast, in the stratified vortex regime in a linearly stratified fluid (see $\mathrm{CJH}$ ) the measured frequency of the density fluctuations decreases with $\Omega$ in the wave regime and then grows linearly with $\Omega$ without any jump in frequency.

Because of the gradual erosion of the pycnocline by the Taylor vortices in the outer homogeneous regions, the parameter $\delta$ (and thus also $N$ ) slowly varies in time and different flow transitions are observed to occur. An example is shown in Fig. 4 for the vortex-vortex regime. Up to the time $T_{1}$ the process is essentially similar to the one depicted in Fig. $2 \mathrm{~b}$, with sinuous oscillations at a certain fundamental frequency. After $T_{2}$, one clearly notices the varying oscillation and the subsequent gradual increase of stochastic noise, apparent from the noise in grey levels in Fig. 4. The described behavior reminds to a classic scenario of transition to chaos via period-doubling cascades (Landau and Lifshitz, 1987).

Since the buoyancy frequency is maximum in the middle of the pycnocline and decreases to zero at its edges, the entrained fluid there has initially less potential energy. Therefore, with the decrease of the pycnocline thickness, the entrainment-rate gradually decreases. Indeed, when scrutinizing the pycnocline edges with time in Fig. 4 one can notice small curvatures.

Fig. 5. (a) Spatio-temporal image of the mixing of a two-fluid system with a sharp pycnocline. The image contains 496 vertical lines taken with acquisition frequency $0.1 \mathrm{~Hz}$; the vertical size of the image is $10 \mathrm{~d}$. (b) Light-intensity profiles $J(z / d)$ at different stages of the mixing process, with time-step $\Delta t=1000 \mathrm{~s}$ between the profiles and solid black lines representing the initial profile at $t=0$. (c) Time-evolution of the normalized potential energy $P$. Experimental parameters: $\epsilon=0.003, \Omega=0.5 \mathrm{rad} / \mathrm{s}, R e=3800$. 


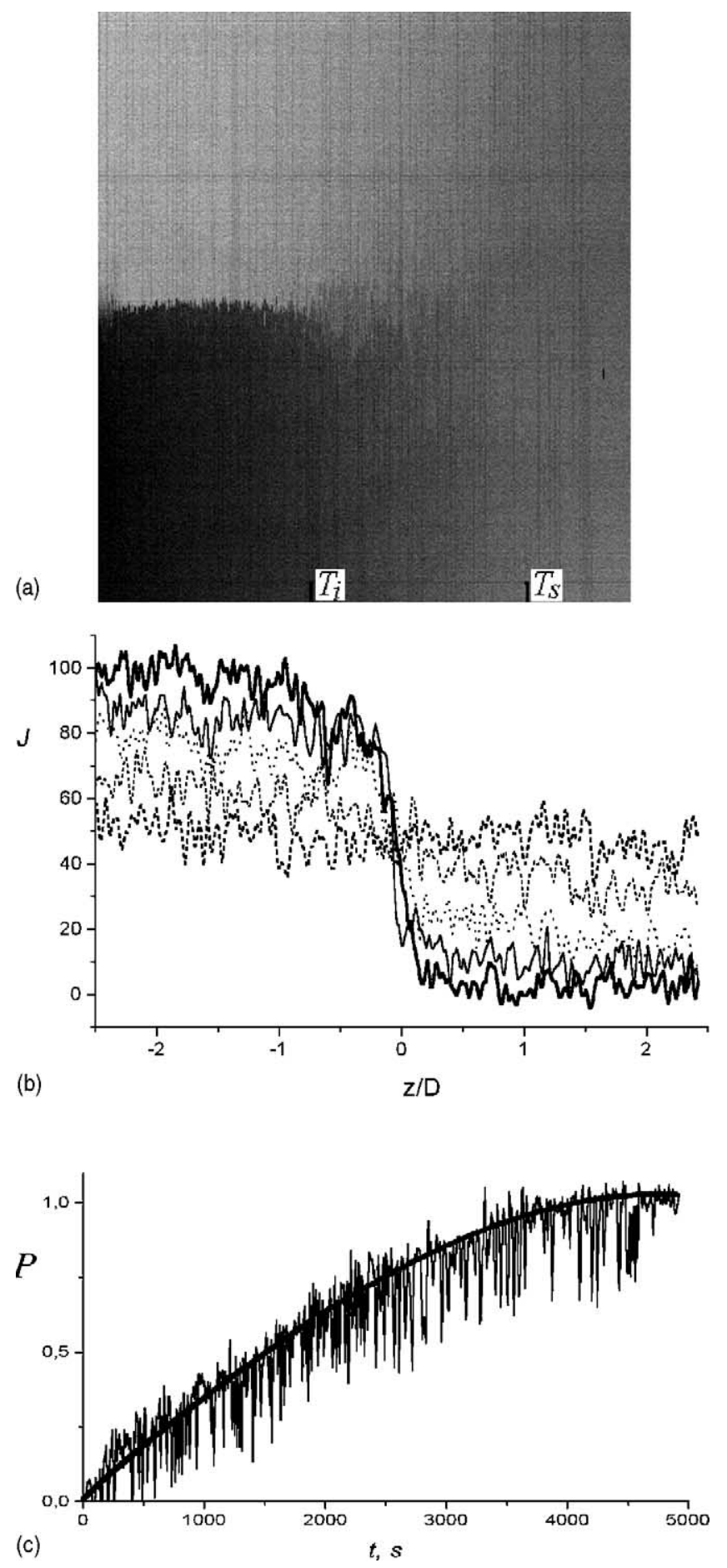




\subsection{Mixing in a two-fluid system}

In this subsection, we focus our attention on the mixing of a two-fluid system with a sharp interface with $\delta / d$ around 0.2 . To keep the duration of experimental runs within reasonable limits, experiments were conducted at Reynolds numbers of 2000 and higher. The flow evolution of which a typical spatio-temporal image of the process is shown in Fig. 5a , was visualized with the laser-induced-fluorescence (LIF) technique. The mixing process can be schematically divided into three stages. In the first stage, the initial density distribution is reduced to a sharp interface with nearly zero $\delta$, as is apparent from the high contrast between light and dark zones in Fig. 5a. In the next stage, entrainment in the upper (and lower) layer of the fluid at the interface gradually decreases the density difference, $\epsilon(t)$, until reaching a critical value $\epsilon_{\mathrm{c}}$ at time $T_{\mathrm{i}}$, when the sharp interface starts to fade. In the subsequent stage the two density fluids mix to one homogeneous layer reached at time $T_{\mathrm{s}}$; after time $T_{\mathrm{s}}$ the light intensity in the image in Fig. 5a has turned uniform grey. These times, $T_{\mathrm{i}}$ and $T_{\mathrm{s}}$ are, respectively, referred to as the interface and stratification life time.

The time-evolution of the light intensity distribution over depth $J(z / D)$ is shown in Fig. 5b. $J$ is normalized such that at $t=0$, its mean initial value in the upper and lower fluid correspond, respectively, to 0 and 100 . Assuming that $J$ is directly proportional to the local density, we can calculate the evolution of the potential energy $P$ of the system with time, which is shown in Fig. 5c in normalized units, with the initial and final levels taken as 0 and 1 . For our experimental setup, the variation of the potential energy between the initial two-fluid and the final homogeneous state corresponds in dimensional form to $\Delta P=\epsilon \rho g \pi\left(b^{2}-a^{2}\right) h^{2} / 2$. We can define the flux Richardson number as the ratio $R i_{\mathrm{f}}=$ $\Delta P / \Delta E$, where $\Delta E$ is the mechanical energy input in the system. The total mechanical energy required to mix the two-layer fluid can be estimated as $\Delta E=M \Omega T_{\mathrm{s}}$, where $M$ is the torque acting on the inner rotating cylinder. We estimated this torque from Dubrulle and Hersant (2002) for a homogeneous fluid, assuming that it is the same as in a stratified fluid. According to Dubrulle and Hersant (2002) the torque is given by $M=G \rho \nu H$, where $G$ is calculated from the gap width and $R e=3800$ according to their relation (20), and gives $G=$ $2.51 \times 10^{6}$. With $\rho=1000 \mathrm{~kg} / \mathrm{m}^{3}, T=1.9 \times 10^{-3} \mathrm{~N} \mathrm{~m}$ and the work performed during the mixing time $T_{\mathrm{s}}=4000 \mathrm{~s}$ the mechanical energy is then $\Delta E=3.82 \mathrm{~J}$. The potential energy variation for two ideally separated layers is $\Delta P=\epsilon \rho_{0} g \pi\left(b^{2}-a^{2}\right) h^{2} / 2$ (where $h=$ $H / 2, \epsilon=0.003)$, which in our case gives $\Delta P=0.117 \mathrm{~J}$, yielding a flux Richardson number, $R i_{\mathrm{f}}=\Delta P / \Delta E=0.031$.

For a fixed rotation rate, i.e., fixed Reynolds number, the variation of $T_{\mathrm{i}}$ and $T_{\mathrm{S}}$ is displayed as a function of the interface density difference, $\epsilon$, in Fig. 6. Both, $T_{\mathrm{i}}$ and $T_{\mathrm{s}}$ are directly proportional to $\epsilon$ implying that the interface and stratification life-times are proportional to the initial potential energy; the mixing efficiency remains thus constant.

For a fixed stratification, $\epsilon$, the dependence of $T_{\mathrm{i}}$ and $T_{\mathrm{s}}$ on $\Omega(\sim R e)$ are shown in Fig. 7 on a logarithmic scale. Within the parameter range under consideration, $T_{\mathrm{i}}$ and $T_{\mathrm{s}}$ are proportional to $\Omega^{-4}$. For the range of $R e$-numbers studied in the present experiments the torque $M \sim R e^{n}$, with $n=3 / 2$ (see Dubrulle and Hersant, 2002). Since the total variation of potential energy $\Delta P$ is the same for all experimental data shown in Fig. 7, we can conclude that $R i_{\mathrm{f}}=\Delta P / \Delta E=1 /\left(M i \Omega T_{\mathrm{s}}\right) \sim R e^{k}$, with $k=3 / 2$. 


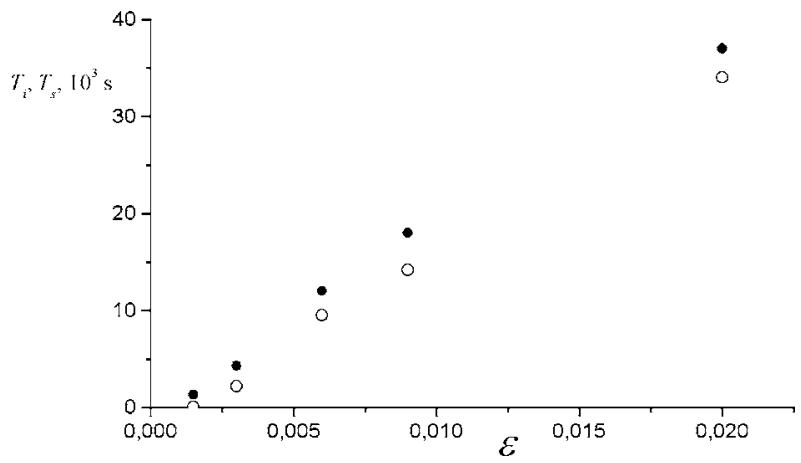

Fig. 6. Values of $T_{\mathrm{i}}$, (open circles) and $T_{\mathrm{s}}$, (black circles), vs. $\epsilon$. The values $T$ are obtained from spatio-temporal shadow-graph images obtained at acquisition frequency of $0.1 \mathrm{~Hz}$. The rotation rate is kept constant at $\Omega=0.5 \mathrm{rad} / \mathrm{s}$ $(\operatorname{Re}=3800)$.

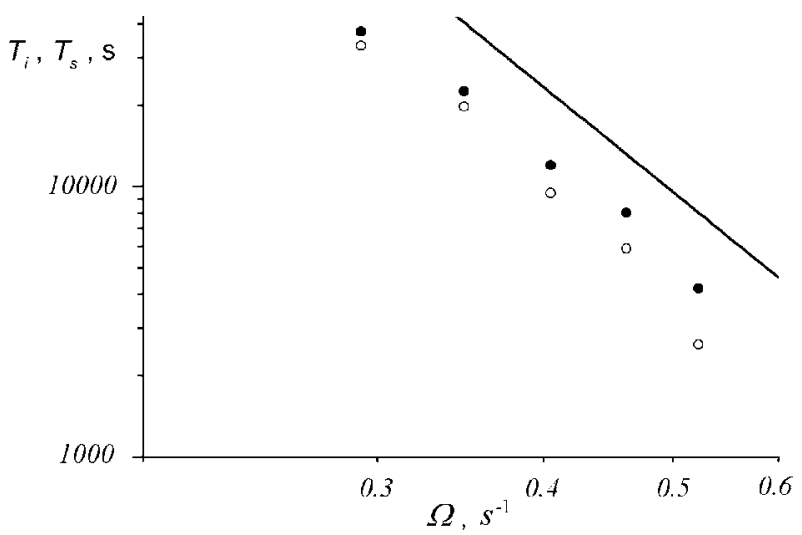

Fig. 7. $\log -\log$ plot of values of $T_{\mathrm{i}}$ (open circles) and $T_{\mathrm{s}}$ (black circles) vs. $\epsilon$. The values $T$ are obtained from spatiotemporal shadow-graph images obtained at acquisition frequency of $0.1 \mathrm{~Hz}$. The stratification of the interface is $\epsilon=0.003$. The solid line has a slope of $\Omega^{-4}$.

\section{Discussion}

Uniform stratification is normally considered as a generic case for the studies on continuously stratified fluids. New dynamical effects occur when the spatial scale of the buoyancy frequency variation is comparable with the characteristic dimension of the flow. In the present study the vertical extension of the pycnocline is comparable with the gap width of the experimental system. We noticed a marked difference in flow evolution with that in a fluid with constant buoyancy frequency. The transition from the wave-vortex to the vortexvortex regime differs significantly from the one described for the uniform stratification in $\mathrm{CJH}$ and is accompanied by a jump of the fundamental frequency of the density fluctuations in the flow. Such a jump implies an increase of the azimuthal mode-number. Physically, the main difference of the present system with the linearly stratified case, is the perturbation 
of the pycnocline by the Taylor vortices in the relatively weakly stratified layers above and below the pycnocline centre. This explains the presence of higher azimuthal modes.

Generally, experiments on mixing in stratified fluids with smooth density profiles (see Fernando, 1991), show a strong tendency toward sharpening of density gradients, with layer formation in case of a thick interface. Depending on the density difference $\epsilon$, an increase of the initial density gradient as well as a decrease of the initial density gradient has been observed.

The mixing efficiency for Reynolds numbers $\mathrm{O}\left(10^{3}\right)$ is found as low as 0.031 compared to values of 0.1 and 0.2 in, respectively, numerical simulations on unstable shear flows (Caulfield and Peltier, 2000) and experiments on turbulence in stratified fluids (Park et al., 1994). The reason must be that the main torque is on the primary azimuthal flow, while the Taylor vortices that mix the flow, are of secondary order. In addition, they are highly anisotropic in contrast to generally investigated turbulent flows. To make a proper comparison with former studies on mixing efficiency, we will need to know the exact strength of the the Taylor vortices; this will be presented in the near future. Nevertheless, these values may be interesting in view of the mixing due to other secondary flows such as for instance Langmuir circulation, which are presently of interest in view of the mixing of the ocean upper layer.

\section{Acknowledgements}

The authors acknowledge the fruitful discussions with Beatrice Janiaud and Emil Hopfinger, and the technical assistance of Pierre Carecchio in setting up the experimental device. Special thanks are due to Bruno Voisin for interesting discussions and steady friendly help. EE was supported by the fellowship MENRT-246 in 2001 which made this research at the LEGI possible and partially by the fund of integration projects SB RAS under grant No. 3.13.1. The experimental work was financed by EPSHOM contact no. 0287028004702925.

\section{References}

Andereck, C.D., Liu, S.S., Swinney, H.L., 1986. Flow regimes in a circular Couette system with independent rotating cylinders. J. Fluid Mech. 164, 155-183.

Boubnov, B.M., Gledzer, E.B., Hopfinger, E.J., 1995. Stratified circular Couette flow: instability and flow regimes. J. Fluid Mech. 292, 333-358.

Boubnov, B.M., Hopfinger, E.J., 1997. Experimental study of circular Couette flow in a stratified fluid. Fluid Dyn. 32, 520-528.

Caton, F., Janiaud, B., Hopfinger, E.J., 1999. Primary and secondary Hopf bifurcations in stratified Taylor-Couette flow. Phys. Rev. Lett. 82, 4647-4650.

Caton, F., Janiaud, B., Hopfinger, E.J., 2000. Stability and bifurcations in stratified Taylor-Couette flow. J. Fluid Mech. 419, 93-124.

Caulfield, C.P., Peltier, W.R., 2000. The anatomy of the mixing transition in homogeneous and stratified free shear layers. J. Fluid Mech. 413, 1-47.

Dubrulle, B., Hersant, F., 2002. Momentum transport and torque scaling in Taylor-Couette flow from an analogy with turbulent convection. Eur. Phys. J. B 26, 379-386.

Fernando, H.J.S., 1991. Turbulent mixing in stratified fluids. Annu. Rev. Fluid Mech. 23, 455-493. 
Garrett, C., 1996. Processes in the surface mixed layer of the ocean. Dyn. Atmos. Oceans 23, 19-34.

Hua, B.L., Le Gentil, S., Orlandi, P., 1997. First transitions in circular Couette flow with axial stratification. Phys. Fluids 9 (2), 365-375.

Kato, H., Phillips, O.M., 1969. On the penetration of the turbulent layer into a stratified fluid. J. Fluid Mech. 37 , 643-665.

Knobloch, E., 1996. Symmetry and instability in rotating hydrodynamic and magnetohydrodynamic flows. Phys. Fluids 8, 1446-1454.

Landau, L.D., Lifshitz, E.M., 1987. Fluid Mechanics. Pergamon Press.

Linden, P., 1973. The interaction of a vortex ring with a sharp density interface: a model for turbulent entrainment. J. Fluid Mech. 60, 467-480.

Linden, P., 1979. Mixing in stratified fluids. Geophys. Astrophys. Fluid Dyn. 26, 379-386.

Park, Y.G., Whitehead, J.A., Gnanadeskian, A., 1994. Turbulent mixing in stratified fluids, layer formation and energetics. J. Fluid Mech. 279, 279.

Staquet, C., Sommeria, J., 2002. Internal gravity waves: from instabilities to turbulence. Ann. Rev. Fluid Mech. 34, 559-593.

Strang, E.J., Fernando, H.J.S., 2001. Entrainment and mixing in stratified shear flows. J. Fluid Mech. 428, 349-386. 